\title{
FIRST KEPLER RESULTS ON RR LYRAE STARS
}

\author{
K. Kolenberg ${ }^{1}$, R. Szabó ${ }^{2}$, D. W. Kurtz ${ }^{3}$, R. L. Gilliland ${ }^{4}$, J. Christensen-DalsgaArd ${ }^{5}$, H. KJeldsen ${ }^{5}$, T. M. Brown ${ }^{6}$, \\ J. M. Benkő ${ }^{2}$, M. Chadid ${ }^{7}$, A. Derekas ${ }^{2,8}$, M. Di Criscienzo ${ }^{9}$, E. GugGenberger ${ }^{1}$, K. Kinemuchi ${ }^{10,11}$, A. Kunder ${ }^{12}$, \\ Z. Kolláth ${ }^{2}$, G. Kopacki ${ }^{13}$, P. Moskalik ${ }^{14}$, J. M. Nemec ${ }^{15}$, J. Nuspl ${ }^{2}$, R. Silvotti ${ }^{16}$, M. D. Suran ${ }^{17}$, W. J. Borucki ${ }^{18}$, \\ D. $\mathrm{KoCH}^{18}$, AND J. M. JENKINS ${ }^{19}$ \\ ${ }^{1}$ Institut für Astronomie, Universität Wien, Türkenschanzstrasse 17, A-1180 Vienna, Austria \\ ${ }^{2}$ Konkoly Observatory, H-1525 Budapest, P.O. Box 67, Hungary \\ ${ }^{3}$ Jeremiah Horrocks Institute of Astrophysics, University of Central Lancashire, Preston PR1 2HE, UK \\ ${ }^{4}$ Space Telescope Science Institute, 3700 San Martin Drive, Baltimore, MD 21218, USA \\ ${ }^{5}$ Department of Physics and Astronomy, Aarhus University, DK-8000 Aarhus C, Denmark \\ ${ }^{6}$ Las Cumbres Observatory Global Telescope, Goleta, CA 93117, USA \\ ${ }^{7}$ Observatoire de la Côte d'Azur, Université Nice Sophia-Antipolis, UMR 6525, Parc Valrose, 06108 Nice Cedex 02, France \\ ${ }^{8}$ Sydney Institute for Astronomy, School of Physics A28, University of Sydney, NSW 2006, Australia \\ ${ }^{9}$ INAF-Osservatorio Astronomico di Roma, Via Frascati 33,00040 Monte Porzio Catone, Rome, Italy \\ ${ }^{10}$ Department of Astronomy, 211 Bryant Space Science Center, University of Florida, Gainesville, FL 32611, USA \\ ${ }^{11}$ Departamento de Astronomía, Universidad de Concepción, Casilla 160-C, Concepcion, Chile \\ ${ }^{12}$ Cerro Tololo Inter-American Observatory, National Optical Astronomy Observatory, La Serena, Chile \\ ${ }^{13}$ Instytut Astronomiczny Uniwersytetu Wrocławskiego, Kopernika 11, 51-622 Wrocław, Poland \\ ${ }^{14}$ Copernicus Astronomical Centre, ul. Bartycka 18, 00-716 Warsaw, Poland \\ ${ }^{15}$ Department of Physics \& Astronomy, Camosun College, Victoria, British Columbia, Canada \\ ${ }^{16}$ INAF-Osservatorio Astronomico di Torino, Strada dell'Osservatorio 20, 10025 Pino Torinese, Italy \\ ${ }^{17}$ Astronomical Institute of the Romanian Academy, Str. Cutitul de Argint, 5, RO 40557, Bucharest, Romania \\ ${ }^{18}$ NASA Ames Research Center, MS 244-30, Moffett Field, CA 94035, USA \\ ${ }^{19}$ SETI Institute/NASA Ames Research Center, MS 244-30, Moffett Field, CA 94035, USA \\ Received 2009 November 15; accepted 2009 December 24; published 2010 March 31
}

\begin{abstract}
We present the first results of our analyses of selected RR Lyrae stars for which data have been obtained by the Kepler Mission. As expected, we find a significant fraction of the RRab stars to show the Blazhko effect, a still unexplained phenomenon that manifests itself as periodic amplitude and phase modulations of the light curve, on timescales of typically tens to hundreds of days. The long time span of the Kepler Mission of $3.5 \mathrm{yr}$ and the unprecedentedly high precision of its data provide a unique opportunity for the study of RR Lyrae stars. Using data of a modulated star observed in the first roll as a showcase, we discuss the data, our analyses, findings, and their implications for our understanding of RR Lyrae stars and the Blazhko effect. With at least $40 \%$ of the RR Lyrae stars in our sample showing modulation, we confirm the high incidence rate that was only found in recent high-precision studies. Moreover, we report the occurrence of additional frequencies, beyond the main pulsation mode and its modulation components. Their half-integer ratio to the main frequency is reminiscent of a period doubling effect caused by resonances, observed for the first time in RR Lyrae stars.
\end{abstract}

Key words: stars: individual: (KIC5559631 (V783 Cyg), KIC3733346 (NR Lyr) and KIC7198959 (RR Lyr)) stars: oscillations - stars: variables: RR Lyrae

Online-only material: color figures

\section{INTRODUCTION}

RR Lyrae stars are low-mass stars that have evolved away from the main sequence and are burning Helium in their core. Their evolutionary stage makes them useful tracers of galactic evolution. Like the Cepheids, they obey a period-luminosity-color relation and are used as distance indicators. RR Lyrae stars have typical periods of $\sim 0.2$ to $\sim 1 \mathrm{~d}$, amplitudes in the optical of 0.3 up to 2 mag, and spectral types of A2 to F6. Most RR Lyrae stars pulsate in the radial fundamental mode (RRab stars), the radial first overtone (RRc stars), and, in some cases, in both modes simultaneously (RRd stars). A few RR Lyrae stars are suspected to be pulsating in higher-order radial overtone modes (Olech \& Moskalik 2009).

\subsection{The Blazhko Effect}

A large fraction of the RR Lyrae stars show a nearly periodic modulation of their light-curve amplitudes and phases on timescales of typically tens to hundreds of days. This so-called Blazhko effect (Blazhko 1907) is one of the most stubborn unsolved problems of the theory of radial stellar pulsations. Since it was discovered more than a century ago, many hypotheses have been proposed to explain the modulation. Over the past decade, focus has predominantly been on resonance models and magnetic models. The oft-quoted resonance models (Van Hoolst et al. 1998; Dziembowski \& Mizerski 2004) involve nonradial modes of low spherical degree (most likely $\ell=1$ ), whereas the magnetic model (Shibahashi 2000) proposes that a strong dipole magnetic field (of the order of $1 \mathrm{kG}$ ) inclined to the rotation axis deforms the main radial mode to have an additional nonradial quadrupole $(\ell=2)$ component aligned with the magnetic field, thus generating the amplitude and phase modulation with the rotation period.

Recently, Chadid et al. (2004) and Kolenberg \& Bagnulo (2009) disproved the presence of a strong magnetic field with dipole-like geometry in RR Lyr, the prototype of the class, and for a sample of RR Lyrae stars, respectively. This implies that 

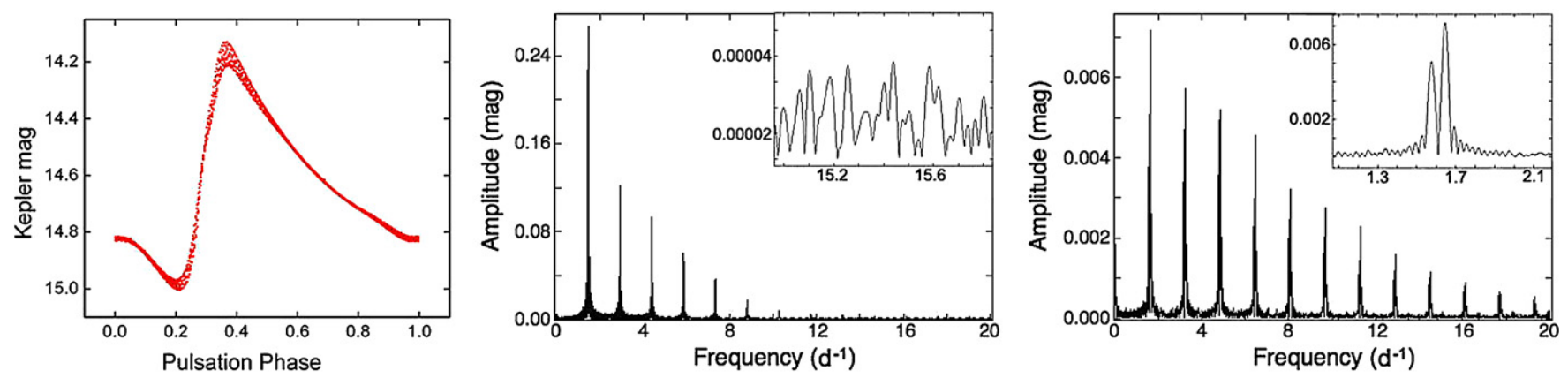

Figure 1. Left: light curve of KIC 5559631 folded with the main period of $0.620699 \mathrm{~d}$. The spread in the width of the light curve is not noise (which is much smaller than the size of the data points) but shows the amplitude and phase modulation over the $27.6 \mathrm{~d}$ Blazhko cycle. Middle: Fourier transform of the raw data converted to the magnitude scale. The inset shows the noise level. Right: Fourier transform after prewhitening with the main frequency and its harmonics. The inset is a zoom, on the same scale, around the position of the main frequency and its harmonics. Triplet components are clearly detected.

(A color version of this figure is available in the online journal.)

the magnetic models in their present form need adaptation, or are not correct. Stothers (2006) proposed a scenario that does not involve nonradial modes, but convective/magnetic interaction as a cause for modulation. The geometry of the magnetic field might be too complex to be detected. Importantly, Jurcsik et al. (2009a) showed that modulated stars change their mean global physical parameters (mean radius, luminosity, and surface effective temperature) over the Blazhko cycle, and phase modulation of the pulsation is interpreted to be a consequence of period changes.

Thus far, none of the proposed models successfully accounts for all of the features observed in modulated RR Lyrae stars. The inspiration for new types of models should come from the observational side. The Kepler Mission will be a prime driver for this.

\subsection{Targets}

To select the RR Lyrae targets for the Kepler Mission, we searched for all known RR Lyrae stars or possible candidates in the Kepler field. The list was compiled using all available variability information contained in the updated catalogs: GCVS (Samus et al. 2002), ASAS North (Pigulski et al. 2009), ROTSE (Akerlof et al. 2000), and HAT (Hartman et al. 2004). KIC10 (KIC, Kepler Input Catalog) was used to get Kepler IDs, coordinates, and magnitudes. The period range 0.15-1.0 days was searched in these catalogs. Light-curve shapes and the $\log P$ versus $J-H$ diagram (Pojmański \& Maciejewski 2004) were also utilized for further selection. Finally, stars with close (bright) companions were excluded. RR Lyr itself, the prototype of the class, is also located in the Kepler field. Despite coordinated ground-based efforts, we were not able to identify any Blazhko star, besides RR Lyr itself, in the Kepler field prior to the launch of the mission.

The Kepler magnitude $K p$ (wide passband between 4300 and $9000 \AA$ ) of the proposed targets lies in the range $7.9 \leqslant$ $K p \leqslant 17.4$. A total of 57 RR Lyrae candidates, including some with uncertain classification, were proposed for observation. Of these, 48 stars were proposed in long cadence (one measurement every 30 minutes) for 90 days and nine stars in short cadence (one measurement every minute) for 30 days. A large fraction ( 23 out of 51) of the stars for which data were released at the time of writing turned out not to be RR Lyrae stars, but, e.g., eclipsing binaries and ellipsoidal variables.

About $60 \%$ of the survey targets will be proposed for continued observations after the survey period.

\section{OBSERVATIONS}

The asteroseismic data released to the KASC (Kepler Asteroseismic Science Consortium) at the time of this writing are long cadence observations. For stars with pulsation cycles of typically half a day this sampling is sufficient to obtain a good coverage of the light curve, but rapid changes and "glitches" in the light curve - as have been reported to occur in some RR Lyrae stars-are missed by this sampling. All of the RR Lyrae stars with Kepler data have so far turned out to be pulsating predominantly in the radial fundamental mode (RRab).

Note that at the time of writing the pipeline for reducing the Kepler data is still being fine-tuned and tested. As a consequence, some low-frequency, low-amplitude variability may be attributable to instrumental effects that will be overcome further into the mission. The results presented in this Letter are valid within this minor constraint.

\section{AN EXAMPLE: A BLAZHKO STAR OBSERVED WITH KEPLER}

To illustrate the potential of Kepler photometry for RR Lyrae stars, we present the first frequency analysis results for the Blazhko star KIC 5559631 (V783 Cyg, see Figure 1). Before the Kepler observations, the star was known to be an RRab star (Samus et al. 2002) with a mean Kepler magnitude of $m_{\mathrm{K}}=$ 14.643 and a period of $P_{0}=0.620699 \mathrm{~d}$, corresponding to a frequency $f_{0}=1.611087 \mathrm{~d}^{-1}$. Blazhko modulation of the light curve was previously unknown. This star was observed in long cadence during the first roll of the Kepler survey phase between HJD 2454964.0109 (2009 May 12) and HJD 2454997.4812 (2009 June 14). (The spacecraft "rolls" every three months to allow for continuous illumination of Kepler's solar arrays. The first such roll, ending the first quarter, occurred on 2009 June 18.) Over the 33.5 day continuous run 1628 data points were obtained for the star. As a consequence of the sampling rate, the Nyquist frequency lies at 24.5 days $^{-1}$.

A small linear trend was removed from the raw data and they were converted to the magnitude scale. We used several frequency analysis software packages, including Period04 (Lenz \& Breger 2005), SigSpec (Reegen 2007), TiFrAn (Csubry 2002), CLEAN (Roberts et al. 1987), and PDM (Stellingwerf 1978).

After prewhitening with the main frequency $f_{0}=1.61117 \pm$ 0.00003 days $^{-1}$ and its significant harmonics (up to $25 f_{0}$; although this is past the Nyquist frequency, it is necessary, as the higher harmonics are detected by their "reflection into the 


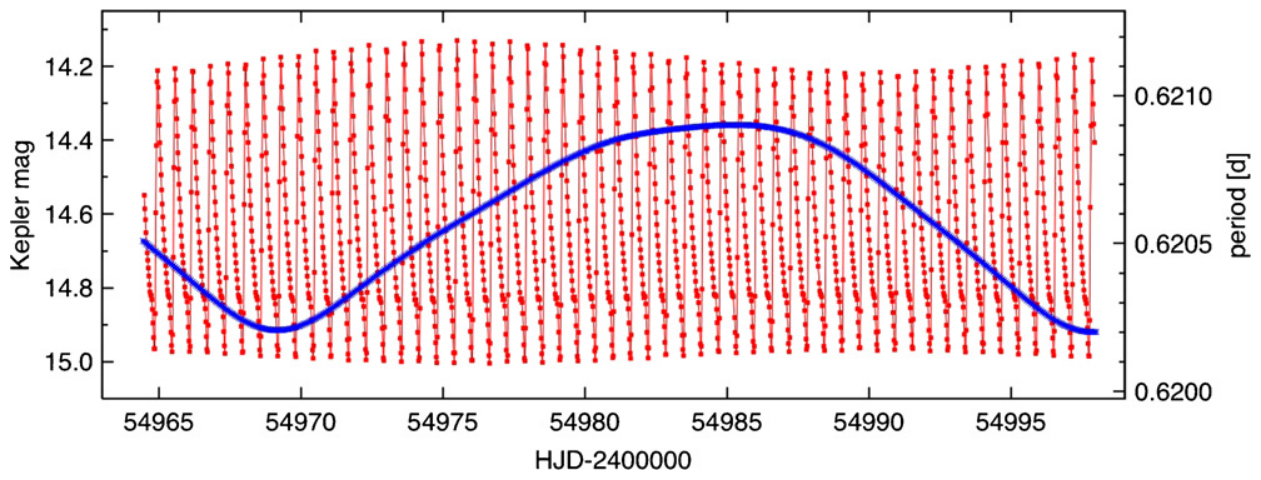

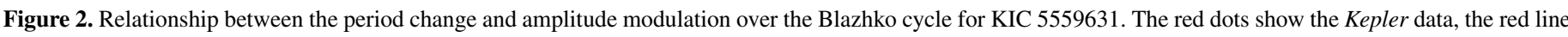

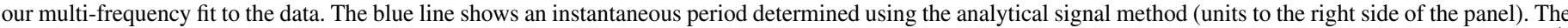

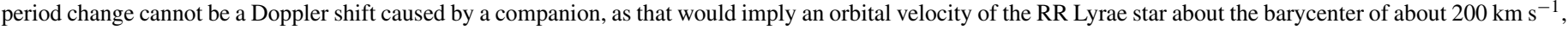
requiring an impossibly large mass for the companion.

(A color version of this figure is available in the online journal.)

range below the Nyquist frequency by beating with the sampling frequency), we clearly see the triplet frequencies that are a typical feature of Blazhko RR Lyrae stars (see Figure 1, right panel). The first side peak appears at $f_{0}+f_{B}=$ 1.64735 days $^{-1}$. This yields an initial value for the Blazhko frequency of $f_{B}=0.0362 \pm 0.0006$ days $^{-1}$ or a Blazhko period of $P_{B}=27.6 \pm 0.5$ days. Subsequent prewhitening reveals additional triplet frequencies equidistantly spaced around the main frequency and its harmonics. The triplet components are detected successively and significant up to the 23 rd order. The Blazhko frequency $f_{B}$ itself is also detected directly from our data of KIC 5559631, though the peak in the Fourier spectrum at its location is very wide due to the short time base of the data. Its detection implies that there is a variation of the mean brightness of the star with the Blazhko cycle. Though modulated RR Lyrae stars are commonly fitted with equidistant multiplet structures, it is worthwhile to test whether there are departures from equidistance. This will be done in our future analyses with a longer time base and hence better frequency resolution.

We fitted the light curve of KIC 5559631 with the main frequency, its harmonics, the significant equidistant triplets, and the Blazhko frequency. The presence of further significant frequencies will be determined on the basis of future data of this target.

Figure 2 shows the light-curve modulation over the Blazhko cycle for KIC 5559631. An instantaneous period was determined continuously by the analytical signal method (Kolláth et al. 2002), shown by the blue line in Figure 2. The period change is non-sinusoidal and there is a considerable phase lag between the period and amplitude modulation. Similar behavior was found by Szabó et al. (2009) in the analysis of the CoRoT RR Lyrae Blazhko stars. This frequency variability will be fully characterized by the long-term Kepler observations and will provide an additional new constraint on seismic models of RR Lyrae stars, since it must be the result of global changes in the star over the Blazhko cycle.

\section{RESULTS FOR THE OTHER RR LYRAE STARS}

For the $28 \mathrm{RRab}$ stars that have been observed by Kepler so far, we find periods in the range $0.43-0.68$ days (main frequencies $f_{0}$ between 1.46 and 2.29 days $^{-1}$ ), with observed amplitudes of the first Fourier component between 0.18 and 0.44 mag.

Figure 3 shows example light curves of non-modulated (KIC 3733346, NR Lyr) and modulated (KIC 7198959, RR Lyr itself) RR Lyrae stars in our sample and the matching Fourier transforms before and after subtraction of the main frequency and its significant harmonics. Note the occurrence of modulation peaks and additional frequencies in the Fourier spectrum of KIC 7198959

Some modulated stars in our sample already clearly show higher-order modulation side lobes in their frequency spectra. Their visibility can be attributed to the degree of amplitude/ phase modulation (Szeidl \& Jurcsik 2009, Benkő et al. 2009) that we will quantify in our studies based on larger data sets.

Besides the modulation components that occur in multiplet structures around the main frequency and its harmonics, we also see additional frequencies that are not at the expected positions of radial modes in RR Lyrae stars (see also Section 5.2). They have significant amplitudes surpassing the mmag level (e.g., 8 mmag in KIC 7198959). Their nature will be investigated on the basis of forthcoming data.

\section{DISCUSSION}

\subsection{Modulation Statistics}

An important statistic that should help constrain models for the Blazhko effect is its occurrence rate. In different stellar populations the relative number of Blazhko stars may vary (see Kovács 2009, and references therein). Previous estimates were that at least $20 \%-30 \%$ of the galactic RRab stars and $5 \%-40 \%$ of the RRc stars (the high RRc incidence rate was found in Omega Cen) are modulated (Szeidl 1988; Moskalik \& Poretti 2003; Mizerski 2003; Moskalik \& Olech 2008). Recent results from the Konkoly Blazhko Survey (Jurcsik et al. 2009b) indicate a $\sim 47 \%$ occurrence rate of the Blazhko effect (14 out of 30 RRab stars). From the excellent CoRoT data an even larger fraction of the observed RR Lyrae stars are modulated, though the sample size is very small (Szabó et al. 2009). Jurcsik et al. (2009b) mention that the significant increase of the incidence rate is a consequence of the discovery of small-amplitude modulation (see, e.g., Jurcsik et al. 2006) that would not have been detected in any previous survey.

In our sample of Kepler RR Lyrae targets, 11 out of 28 RRab stars are clearly modulated (with large amplitude) and for a few more we suspect small amplitude modulation. Though at this point our numbers are merely indicative, we obtain an incidence rate of at least $40 \%$, in agreement with the findings from recent high-precision studies (Jurcsik et al. 2009b), and higher than previously thought. Is every RRab star modulated? 

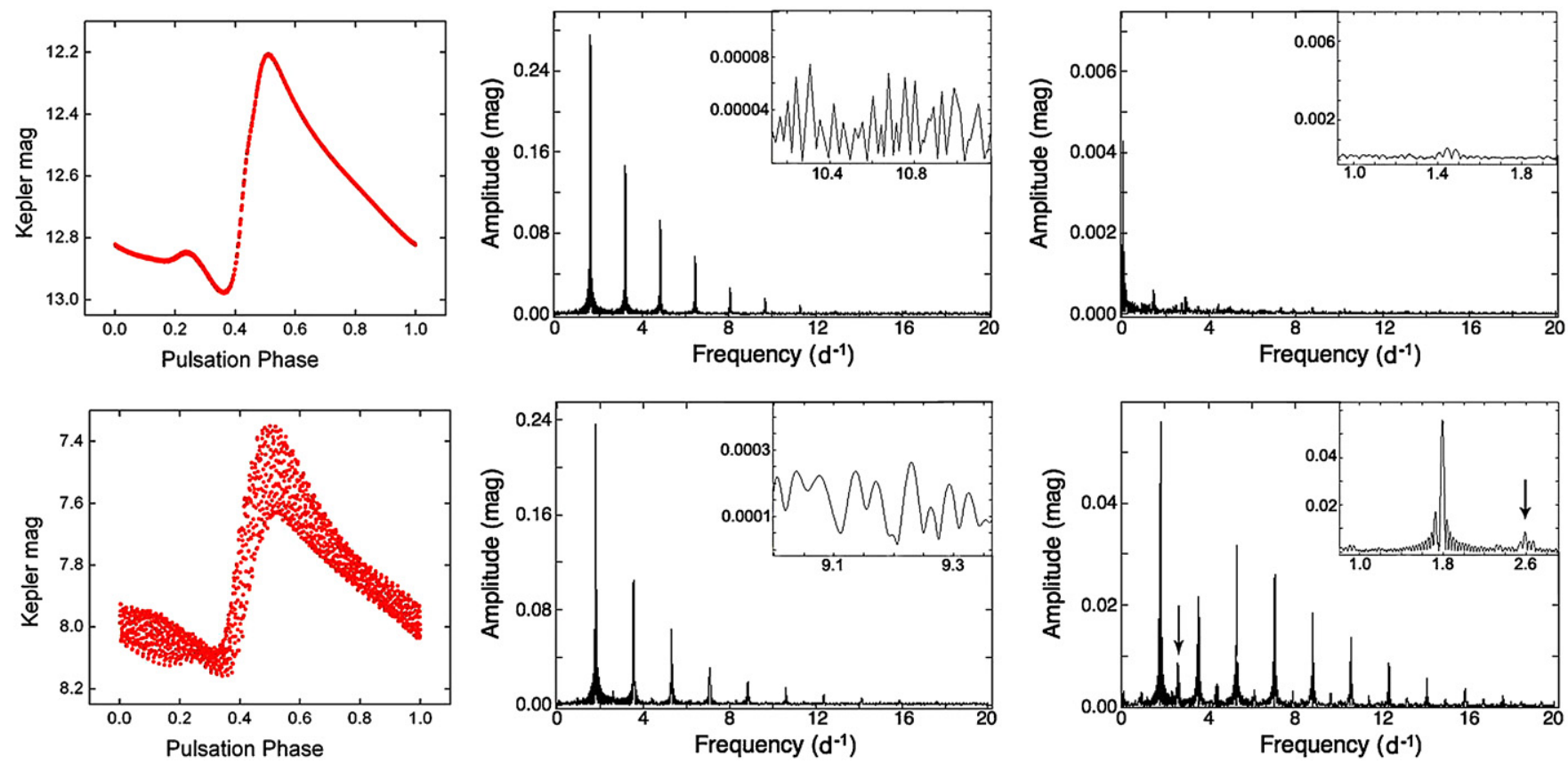

Figure 3. Left: light curves of two Kepler RR Lyrae stars (KIC 3733346 and KIC 7198959) folded with the main period. Middle: Fourier transform of the raw data converted to the magnitude scale. The inset shows the noise level. Right: Fourier transform after prewhitening with the main frequency and its harmonics. The inset, on the same scale, is a zoom around the position of the main frequency and its harmonics. For the modulated star (KIC 7198959) multiplet components are clearly detected. Arrows point at the highest peak connected to an additional frequency.

(A color version of this figure is available in the online journal.)

What fraction of RR Lyrae stars are modulated to within certain detection limits? With unprecedented precision and the longer Kepler data sets we will investigate these questions, too.

\subsection{Additional Frequencies}

For a few of our targets, we detect additional frequencies in our Kepler data that are not at the expected positions of the radial modes in RR Lyrae stars. However, they appear at frequency ratios close to $3 / 2,1 / 2,5 / 2,7 / 2$, with the radial fundamental mode $f_{0}$ (Figure 4 ). These ratios are reminiscent of resonance phenomena, specifically period doubling bifurcation as described by Moskalik \& Buchler (1990) and Buchler \& Moskalik (1992) for Cepheid and BL Her models (see also Figure 3 in Moskalik \& Buchler 1991, for comparison with our Figure 4). Such period doubling would also result in alternating heights of maxima, as we observe in these targets (Figure 4).

In his recent review of the Blazhko effect, and our (lack of) understanding of the phenomenon, Kovács (2009) stresses that more attention should again be paid to models based on radial mode resonances (see, e.g., Goranskij et al. 2009). After the models by Borkowski (1980) and Moskalik (1986) were dismissed by lack of confirmation from hydrodynamical models, most attention has been on resonances involving nonradial modes.

In several recent studies of RR Lyrae stars additional frequencies were detected, e.g., in photometry of the RRd star AQ Leo (Gruberbauer et al. 2007) from the MOST satellite and in the V1127 Aql data from the CoRoT mission (Chadid et al. 2010). These frequencies were not found at positions where radial modes or radial resonances would occur. They may, therefore, be connected to nonradial modes. Nonradial modes play a crucial role in several models for explaining the Blazhko modulation (e.g., Dziembowski \& Mizerski 2004). Their presence may, however, be unrelated to the Blazhko effect. Despite several

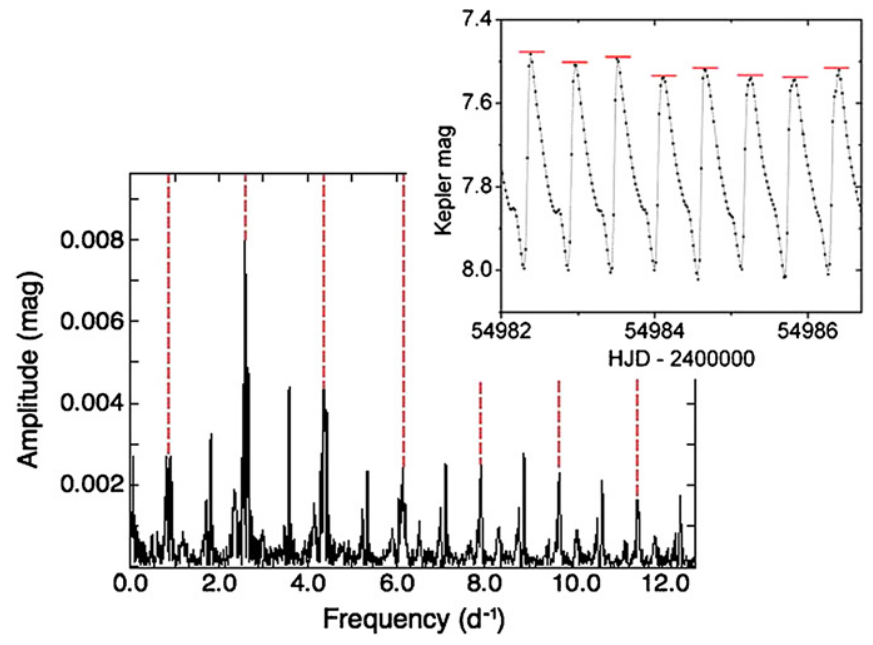

Figure 4. Residual spectrum of KIC 7198959 after subtraction of the main frequency $f_{0}$, its harmonics, and the triplet components. The dashed lines mark the positions of the half-integer frequency components. The highest peak occurs at $\sim 3 / 2 f_{0}$. The frequency peaks in between the dashed lines are mostly higherorder multiplet components present in the spectrum around $f_{0}$ and its harmonics. We did not subtract these yet, as the Blazhko period of KIC 7198959 is only partially covered by these data. The inset shows the alternating heights of the maxima around minimum light for the star, connected to the additional frequencies.

(A color version of this figure is available in the online journal.)

claims (Olech et al. 1999; Chadid et al. 1999) of detection and attempts (Kolenberg 2002) at their identification, there has been no unambiguous proof of nonradial modes in RR Lyrae stars so far. We hope that through the ultra-precise data from the Kepler Mission we will be able to obtain certainty about their existence. Confirmation and exploration of nonradial pulsation would be extremely important to shift the current radial (one-dimensional) 
theoretical modeling to a nonradial (multi-dimensional) hydrodynamical description of RR Lyrae pulsations.

\subsection{Quality and Advantages of Kepler Photometry}

From the first releases of Kepler data, it is obvious that Kepler photometry is stable enough to be tied to ground-based photometry, even if data are not taken simultaneously. Ground-based follow-up photometry and spectroscopy of selected Kepler targets is underway to improve the information in the Kepler Input Catalog. High-resolution multi-color single-epoch imaging of the targets is important to mitigate the 4 arcsec sampling of the Kepler photometer. The latter may cause some problems concerning proper identification, determination of the total pulsational amplitude, and blending.

Although the CoRoT satellite is carrying out a similar mission to Kepler, and MOST is also delivering valuable results in asteroseismology, Kepler has significant advantages for the study of RR Lyrae stars. These include the large aperture of the primary mirror: $95 \mathrm{~cm}$ diameter (versus $28 \mathrm{~cm}$ for CoRoT and $15 \mathrm{~cm}$ for MOST) allowing the observation of fainter stars and higher precision photometry, the potential for obtaining observations over a longer time base: $3.5-5$ yr (versus a maximum of $150 \mathrm{~d}$ for CoRoT and about 8 weeks for MOST in order to cover also longer cycles for modulated RR Lyrae stars (e.g., RR Lyr's 4 yr cycle), and the larger field of view: $105 \mathrm{deg}^{2}$ which allows the observation of a significantly large sample of RR Lyrae stars simultaneously. Finally, thanks to Kepler's Earth-trailing orbit (versus CoRoT's and MOST's lowEarth orbits), Kepler is spared from transits through the South Atlantic Anomaly.

\section{SUMMARY}

The first releases of Kepler data have yielded light curves with unprecedented quality. In this Letter, we have attempted to illustrate the tremendous potential of Kepler for studies of RR Lyrae stars.

The major results and prospects for further analysis can be summarized as follows.

1. The fraction of modulated stars in our sample of 28 observed stars so far confirms that the Blazhko effect is much more common than previously thought (see also Jurcsik et al. 2009b). We can already fix a lower limit of $\sim 40 \%$ for the incidence rate in our sample. The Kepler data will help to determine a lower limit for the modulation amplitude and to constrain the models.

2. We observe additional frequencies, beyond the main frequency and classically expected modulation components, in the frequency spectra of a number of modulated RR Lyrae stars. Their ratio to the main frequency suggests resonance effects, in particular a period doubling bifurcation as described by Moskalik \& Buchler (1990). It is for the first time that such frequencies are reported in RR Lyrae stars.

3. The star KIC 5559631 (V783 Cyg) presented in this Letter has its modulation cycle (27.6 days) covered by the Q1 data. For the other Blazhko stars the modulation periods are longer. Forthcoming data with a longer time base will allow for a multitude of analyses: besides the classical ways of analyzing Blazhko stars (Fourier techniques, phase dispersion minimization), we will investigate the relative contribution of phase and amplitude modulation in RR Lyrae stars (Szeidl \& Jurcsik 2009; Benkő et al. 2009).

4. In our forthcoming analyses, we will search for second and higher overtone (radial) modes in the time-series photometry, as well as potential nonradial modes.

5. Several authors (LaCluyzé et al. 2004; Kolenberg et al. 2006; Jurcsik et al. 2009b; Clement \& Goranskij 1999; Goranskij et al. 2009) have found that the modulation over the Blazhko cycle can be very unstable. The long time base of the Kepler data will allow us to study the stability of pulsation and modulation with unprecedented precision. The Kepler data also have the required precision to investigate irregularities in the pulsation of non-modulated RR Lyrae stars.

6. We will also investigate features connected to shock wave phenomena, such as the change in slope at mid-rising light and the amplitudes and durations of the hump and bump (see Gillet \& Crowe 1988) in both stable RRab stars and during Blazhko cycles.

7. Theoretically predicted strange mode pulsation (Buchler \& Kolláth 2001), which has not been observed in RR Lyrae stars yet, may also be present in the data.

8. Further possible applications of the 3.5-5 yr Kepler data are the measurement of the secular variation of the pulsation period(s), directly linked with the evolutionary status of a star (see, e.g., Poretti et al. 2008), and the detection of substellar companions with the timing method, as was done with another class of horizontal branch pulsators (e.g., Silvotti et al. 2007).

Funding for this Discovery mission is provided by NASA's Science Mission Directorate. The authors thank the entire Kepler team without whom these results would not be possible. K. K. and E. G. are supported by the Austrian Research Fund (FWF) project T359 and P19962. R. Sz., J. B., Z. K., and J. N. acknowledge the financial support of KvVM-MUI grant No. K-36-08-00031K. D. W. K. acknowledges support by the UK Science and Technology Facilities Council.

Facilities: Kepler

\section{REFERENCES}

Akerlof, C., et al. 2000, AJ, 119, 1901

Benkő, J., et al. 2009, in AIP Conf. Proc. 1170, Stellar Pulsation: Challenges for Theory and Observation, ed. J. A. Guzik \& P. A. Bradley (Melville, NY: AIP), 273

Blazhko, S. N. 1907, Astron. Nachr., 175, 325

Borkowski, K. J. 1980, Acta Astron., 30, 393

Buchler, J. R., \& Kolláth, Z. 2001, ApJ, 555, 961

Buchler, J. R., \& Moskalik, P. 1992, ApJ, 391, 736 (Section 3.5)

Chadid, M., Kolenberg, K., Aerts, C., \& Gillet, D. 1999, A\&A, 352, 201

Chadid, M., Wade, G. A., Shorlin, S. L. S., \& Landstreet, J. D. 2004, A\&A, 413, 1087

Chadid, M., et al. 2010, A\&A, in press

Clement, C., \& Goranskij, V. P. 1999, ApJ, 513, 767

Csubry, Z. 2002, in 2nd Workshop of Young Researchers in Astronomy and Astrophysics 12, ed. E. Forgács-Dajka (Publ. Astron. Dep. Eötvös Loránd Univ.), 117

Dziembowski, W. A., \& Mizerski, T. 2004, Acta Astron., 54, 363

Gillet, D., \& Crowe, R. A. 1988, A\&A, 199, 242

Goranskij, V. P., Clement, C., \& Thompson, M. 2009, in Proc. of the Kukarkin Centenary Meeting, ed. C. Sterken, et al., in press

Gruberbauer, M., et al. 2007, MNRAS, 379, 1498

Hartman, J. D., et al. 2004, AJ, 128, 1761

Jurcsik, J., Szeidl, B., \& Sódor, Á. 2006, AJ, 132, 61

Jurcsik, J., et al. 2009a, MNRAS, 393, 1553 
Jurcsik, J., et al. 2009b, MNRAS, 400, 1006

Kolenberg, K. 2002, PhD thesis, Univ. of Leuven, Belgium

Kolenberg, K., \& Bagnulo, S. 2009, A\&A, 498, 543

Kolenberg, K., et al. 2006, A\&A, 459, 577

Kolláth, Z., Buchler, J. R., Szabó, R., \& Csubry, Z. 2002, A\&A, 385, 932

Kovács, G. 2009, in AIP Conf. Proc. 1170, Stellar Pulsation: Challenges for Theory and Observation, ed. J. A. Guzik \& P. A. Bradley (Melville, NY AIP), 261

LaCluyzé, A., et al. 2004, AJ, 127, 1653

Lenz, P., \& Breger, M. 2005, Commun. Asteroseismol., 146, 53

Mizerski, T. 2003, Acta Astron., 53, 307

Moskalik, P. 1986, Acta Astron., 36, 333

Moskalik, P., \& Buchler, J. R. 1990, ApJ, 355, 590

Moskalik, P., \& Buchler, J. R. 1991, ApJ, 366, 300

Moskalik, P., \& Olech, A. 2008, Commun. Asteroseismol., 157, 345

Moskalik, P. \& Poretti, E. 2003, A\&A, 398, 213

Olech, A., Kałużny, J., \& Thompson, I. B. 1999, AJ, 118, 442

Olech, A., \& Moskalik, P. 2009, A\&A, 494, L17

Pigulski, A., et al. 2009, Acta Astron., 59, 33
Pojmański, G., \& Maciejewski, G. 2004, Acta Astron., 54, 153

Poretti, E., et al. 2008, Mem. Soc. Astron. Ital., 79, 471

Reegen, P. 2007, A\&A, 467, 1353

Roberts, D. H., Lehar, J., \& Dreher, J. W. 1987, AJ, 93, 968

Samus, N. N., et al. 2002, Astron. Lett., 28, 174

Shibahashi, H. 2000, in ASP Conf. Ser. 203, The Impact of Large-Scale Surveys on Pulsating Star Research, ed. L. Szabados \& D. Kurtz (San Francisco, CA: ASP), 299

Silvotti, R., et al. 2007, Nature, 449, 189

Stellingwerf, R. F. 1978, ApJ, 224, 953

Stothers, R. B. 2006, ApJ, 653, 73

Szabó, R., et al. 2009, in AIP Conf. Proc 1170, Stallar Pulsation: Challenges for Theory and Observation, ed. J. A. Guzik \& P. A. Bradley (Melville, NY: AIP), 291

Szeidl, B. 1988, in Proc. Workshop Budapest 1987, Multimode Stellar Pulsations, ed. G. Kovacs, L. Szabados, \& B. Szeidl (Budapest: Konkoly Observatory, Kultura), 45

Szeidl, B., \& Jurcsik, J. 2009, arXiv:0906.3987

Van Hoolst, T., Dziembowski, W. A., \& Kawaler, S. D. 1998, MNRAS, 297, 536 